\title{
Continuous decline in prevalence of drug resistance mutations among naïve and CART experienced HIV-positive patients in Poland over time

\author{
GP Stanczak*1, KJ Przybylska-Stengiel1, E Firlag-Burkacka1, A Wiercinska- \\ Drapalo $^{2}$, A Horban ${ }^{1}$ and JJ Stanczak ${ }^{1}$
}

Address: ${ }^{1}$ Hospital for Infectious Diseases, Warsaw, Poland and ${ }^{2}$ Medical University, Bialystok, Poland

* Corresponding author

from Ninth International Congress on Drug Therapy in HIV Infection

Glasgow, UK. 9-13 November 2008

Published: 10 November 2008

Journal of the International AIDS Society 2008, I I (SuppI I):PI84 doi:I0.II86/I758-2652-I I-SI-PI84

This abstract is available from: http://www.jiasociety.org/content/II/SI/PI84

(c) 2008 Stanczak et al; licensee BioMed Central Ltd.

\section{Purpose of the study}

Current guidelines recommend drug resistance (DR) testing before the initiation of therapy. The aim of this study was to assess the prevalence of DR mutations among newly infected and cART experienced persons in Poland and compare them with the values obtained in the years 2006 and 2002-2005.

\section{Methods}

The sequencing of protease and part of reverse transcriptase region was performed using Trugene or ViroSeq assay. The prevalence of drug resistance mutations was estimated according to mutation list created for epidemiological estimates of transmitted resistance [1]. Class resistance was defined as the presence of at least one mutation associated with resistance to any drug in that class.

\section{Summary of results}

The study included 103 treatment naïve and 123 were cART experienced persons. In the treatment naïve group, the prevalence of transmitted DR was 3.9\% (5.8\% in 2006 and $14.1 \%$ in 2002-2005) and in all cases limited to single classes. Mutations associated with resistance to NRTIs and to NNRTIs were present in $1.9 \%$ of cases each. No transmitted resistance to PIs was detected. In the cART experienced group, the prevalence of DR mutations reached $43.1 \%(52.6 \%$ in 2006 and $76.7 \%$ in $2002-$ 2005). Most of the virus variants were resistant to two classes of drugs (28\%), followed by resistance to one class $(13 \%)$. In two cases a three-class resistant strain was detected $(1.6 \%)$. The most common were mutations associated with resistance to NRTIs (38\%), followed by NNRTIs (24\%) and PIs (15\%).

\section{Conclusion}

The prevalence of DR mutations in both analyzed groups decreased when compared to previous studies from 2006 and 2002-2005. It may be the effect of more effective antiretroviral drugs, incorporation of GT results into clinical practice, and/or growing awareness of the role of adherence. Transmission of DR mutations in Poland is limited to single classes which is concordant with the results of a study conducted by the SPREAD programme in Europe [2]. The publication of standardized lists of HIV-1 protease and reverse transcriptase mutations for DR surveillance [1] facilitates the comparison of results obtained in different countries.

\section{References}

I. Shafer, et al:: HIV-I protease and reverse transcriptase mutations for drug resistance surveillance. AIDS 2007, 2 I:2 I 5-23.

2. Transmission of drug-resistant HIV-I in Europe remains limited to single classes. The SPREAD progamme. AIDS 2008, 22:625-635. 